\title{
Case Report \\ Confluent Small Bowel Lipomatosis: A Rare Cause of Recurrent Abdominal Pain
}

\author{
Sukhwant Khanijaun ${ }^{1,2}(\mathbb{D})$, Rajkumar Cheluvappa ${ }^{3}(\mathbb{D}$, Selwyn Selvendran 4 (D) and Tony Pang $1,2,5, *(\mathbb{D})$ \\ 1 Department of Surgery, Westmead Hospital, Westmead, NSW 2145, Australia; sukhsk@gmail.com \\ 2 Faculty of Medicine and Health, Westmead Clinical School, The University of Sydney, \\ Westmead, NSW 2145, Australia \\ 3 Nursing and Midwifery, Australian Catholic University, Watson, ACT 2602, Australia; \\ rajkumarchel@gmail.com \\ 4 Department of Surgery, St George Hospital, Kogarah, NSW 2217, Australia; tselvendran@hotmail.com \\ 5 Surgical Innovations Unit, Westmead Hospital, Westmead, NSW 2145, Australia \\ * Correspondence: tony.pang@sydney.edu.au; Tel.: +61-8890-3537
}

Citation: Khanijaun, S.; Cheluvappa, R.; Selvendran, S.; Pang, T. Confluent Small Bowel Lipomatosis: A Rare Cause of Recurrent Abdominal Pain. Surgeries 2022, 3, 11-17. https:// doi.org/10.3390/surgeries3010003

Academic Editor: Cornelis F. M. Sier

Received: 29 December 2021

Accepted: 22 January 2022

Published: 26 January 2022

Publisher's Note: MDPI stays neutral with regard to jurisdictional claims in published maps and institutional affiliations.

Copyright: (C) 2022 by the authors. Licensee MDPI, Basel, Switzerland. This article is an open access article distributed under the terms and conditions of the Creative Commons Attribution (CC BY) license (https:// creativecommons.org/licenses/by/ $4.0 /)$.

\begin{abstract}
Small intestine lipomatosis is rare but may be associated with pain, intussusception, and gastrointestinal bleeding. In this report, we examine the case of a 41-year-old man who had recurrent presentations to the emergency department with non-specific abdominal pain. Preoperative imaging suggested extensive infiltration of small intestine with macroscopic fat. At surgery, extensive and confluent small bowel lipomatosis were seen. The affected ileal segment was resected, and the patient remained symptom-free after surgery. Abdominal lipomatosis is a rare condition which can be completely treated by resection of the affected gut segment but is often unsuspected and difficult to diagnose. In this report, we describe a case with the most extensive lipomatosis on record with more than $70 \mathrm{~cm}$ of gut with confluent lipomatosis. Magnetic resonance Enterography (MRE) is a useful non-invasive diagnostic modality, although laparoscopy/laparotomy may be necessary for assessment of the extent of disease. Symptomatic cases should be treated with segmental small bowel resection, which is curative.
\end{abstract}

Keywords: abdominal pain; intussusception; lipoma; lipomatosis; magnetic resonance enterography; pain; resection; small intestine

\section{Introduction}

Small intestinal lipomatosis is a rare condition which may present with non-specific abdominal pain [1-14]. At autopsy, the incidence of small bowel lipomas is estimated to be from 0.04 to $5.8 \%$ [4,15-18]. The most common complication of intestinal lipomas is small bowel intussusception, frequently at the ileocaecal junction, caused by a lipoma becoming a focal lead point $[1,3-6,8,10,18]$. Small bowel lipomas rarely cause bleeding $[2,19,20]$, intestinal obstruction [21,22], or iron deficiency anaemia [23,24]. A case of lipoma associated with bowel perforation has also been reported [18].

Submucosal lipomas of the gastrointestinal tract are solitary in 85 to $98 \%$ of cases [19]. While such solitary small bowel submucosal lipomas are not uncommon, very few cases of multiple small bowel lipomatosis have also been reported [11-14,25-28]. Rarer yet are confluent lipomatosis, such as in the case described below. When presenting with nonspecific clinical features and minimal endoscopic findings, extensive lipomatosis poses a significant diagnostic challenge, as described in our case below.

\section{Case Details}

A 41-year-old man initially presented to the emergency department of our hospital with a 2-week history of episodic central abdomen pain. His history of presenting illness included $2 \mathrm{~h}$ of burning and stabbing pain radiating inferiorly and to the back. The 
pain was mostly persistent but presented with colicky exacerbations. His episodic pain was precipitated by food, starting a few hours after ingestion. Avoiding solid food and maintaining a fluid diet appeared to partially ameliorate his pain. He did not have nausea, vomiting, or variations in his bowel habits.

The patient's past medical history was unremarkable with the consumption of 2 to 3 standard drinks every day. He recently recommenced smoking after abstaining for 13 years, attributing this to personal issues. In the last 2 months, the patient had lost $9 \mathrm{~kg}$ of body weight but had no other systemic symptoms. There are doubts with regards to the patient's narrative of a history of Crohn's disease in a cousin, but he reported no other family history of gastrointestinal or haematological diseases.

The patient's vital signs, clinical examination, and blood tests were normal. Specifically, he had no evidence of lymphadenopathy, abdominal masses, abdominal tenderness, or testicular abnormalities. He was given a provisional diagnosis of gastritis and was sent home with pantoprazole only. He was also asked to report to the hospital later to undergo a CT scan and an elective gastroscopy.

Unfortunately, he re-presented to the emergency department two weeks later with continuous abdominal pain, prompting the performance of a CT scan. The CT scan demonstrated two major abnormalities: mural thickening of the small intestine (Figure 1A) and extensive mesenteric lymphadenopathy (Figure 1A,B). Imaging of his head, neck, and chest demonstrated no other areas of lymphadenopathy. As the terminal ileum was suspected to be involved, a colonoscopy was performed to examine this area and to obtain tissue diagnosis. Biopsies of the terminal ileum and caecum returned with findings of benign lymphocytic infiltration.

The patient re-presented multiple times to the emergency department with similar complaints. He had now been having symptoms for around 6 weeks. Further investigation with Magnetic Resonance Enterography (MRE), especially Fast Imaging Employing Steady-state Acquisition (FIESTA) sequences (balanced steady-state gradient echo sequence) demonstrated reduced number of ileal loop folds, copious macroscopic fat within the wall of the small intestine, and what appeared to be diverticula within the small intestine (Figure 1C,D). Most of the small intestine appeared to be to be involved.

Given the primary pathology was clearly identified as small intestinal, enteroscopies were performed to determine and define the extent of disease and to possibly obtain biopsy specimens for histopathological examination. Initially, capsule endoscopy was performed, showing what appeared to be possible areas of mucosal ulceration. Subsequently, antegrade and retrograde balloon enteroscopies were carried out but did not demonstrate clearcut mucosal abnormalities. A CT-guided biopsy of the mesenteric lymph nodes was inconclusive.

Finally, with continued symptoms and diagnostic uncertainty, a laparotomy was performed. An open, rather than laparoscopic approach was taken, as an accurate assessment of the nature and extent of disease was felt to be of vital importance. During surgery, a grossly thickened and dilated ileal segment was evident. It was approximately $150 \mathrm{~cm}$ in length and was contiguous with $50 \mathrm{~cm}$ of normal-looking terminal ileum distal to it. In contrast to the MRE impression, most of the small intestine appeared normal on gross examination. The significant size difference (thickness and dilation) between the normal and abnormal intestinal segments probably accounted for the prior overestimation (length) of involvement. The proximal jejunum/ileum, approximately $440 \mathrm{~cm}$, appeared to be normal. The rest of the abdomen appeared normal including the appendix. 


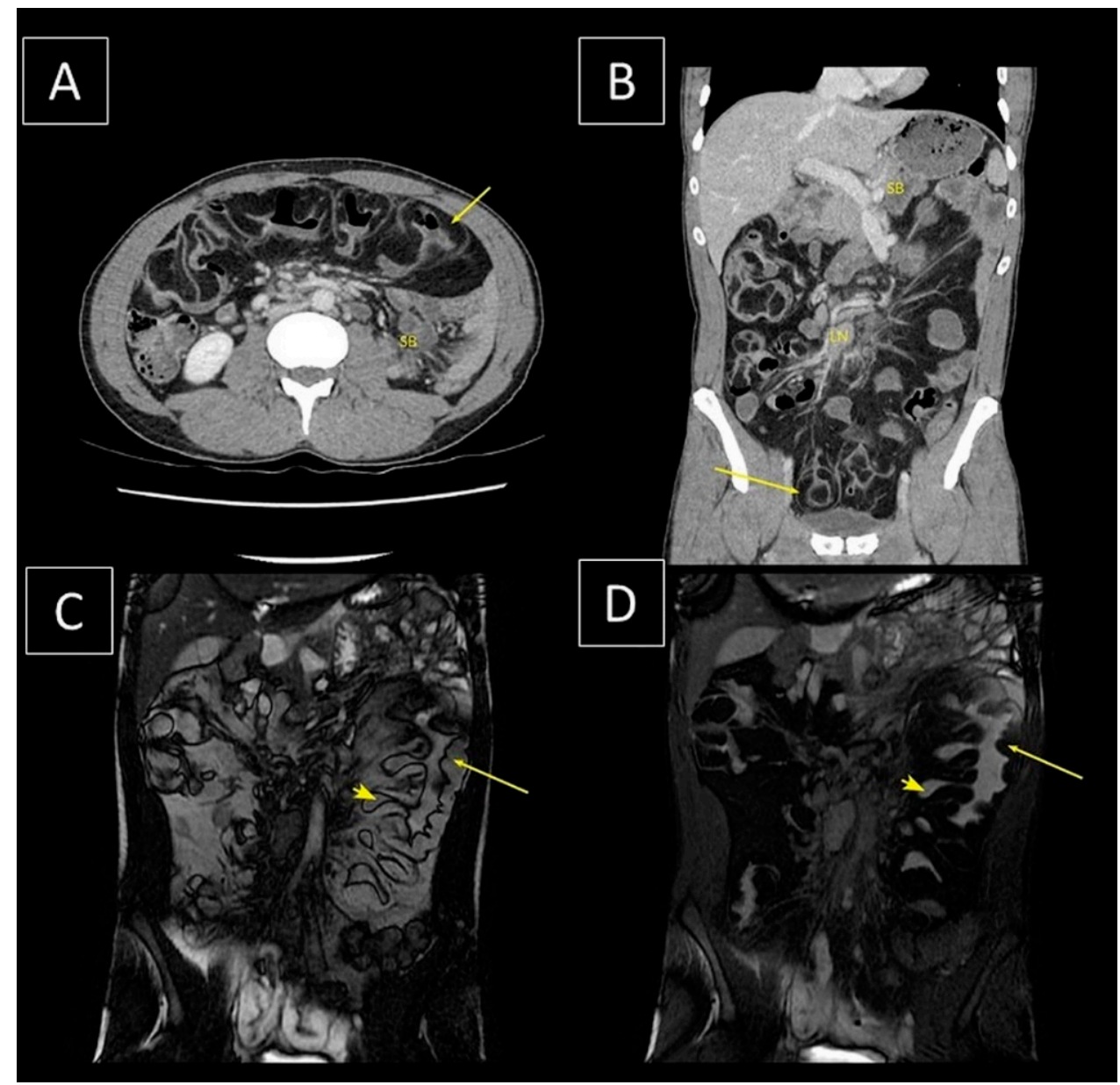

Figure 1. (A) Preoperative Axial CT with portal venous phase intravenous contrast and oral contrast demonstrating thickening of the ileal loops (arrow) and small bowel loops with no mural thickening (SB). The thickened ileal loops demonstrate similar attenuation values as the adjacent mesenteric fat, suggestive of infiltration by fat. (B) Coronal images from the same CT demonstrating submucosal fatty infiltration of ileal loops (arrow), small bowel loops with no mural thickening (SB), and mesenteric lymphadenopathy (LN). (C,D) Corresponding coronal images from the FIESTA sequences (balanced steady-state gradient echo sequence) of the MR enterography. Loss of signal in the small bowel mural thickening (arrows) on the fat-suppressed FIESTA image (D) suggests that the infiltrative material is macroscopic fat. The arrowhead demonstrates one of the numerous diverticula in the involved small bowel.

In the drainage area of the thickened and dilated ileal segment, mesenteric lymphadenopathy was obvious with the apical node being the largest lymph node. The abnormal segment of small intestine and the abnormal mesenteric lymph nodes were resected, and a functional end-to-end (side-to-side) stapled anastomosis was performed. The length of the resected intestinal segment was $150 \mathrm{~cm}$, inclusive of more than $70 \mathrm{~cm}$ of ileum with lipomas (Figure 2A). When the resected gut specimen was dissected via a longitudinal incision, the mucosa appeared thickened and polypoid over the coalescing accumulation of lipomas (Figure 2B). The mucosa also displayed occasional ulceration, diverticula, and puckering (Figure 2B). The lipomas ranged from $0.1 \mathrm{~cm}$ to $13 \mathrm{~cm}$ each in diameter and presented as mural thickening composed of bulging yellow fat (Figure 2B). Although the lipomas were individually discernible and circumscribed, they were so numerous as to appear confluent (Figure 2B). 


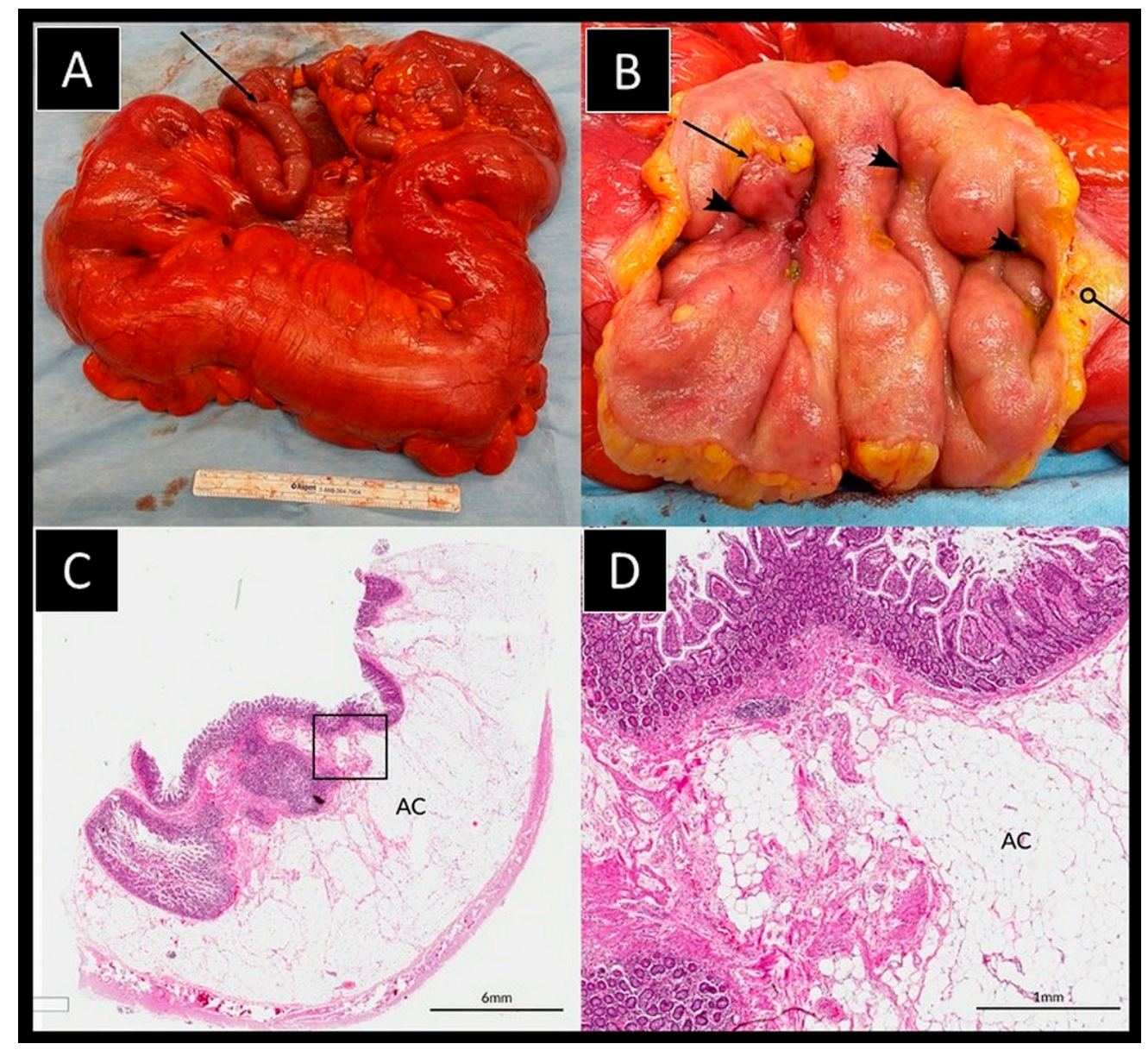

Figure 2. (A) Gross morphology (macroscopic appearance) of the resected segment of thickened and dilated ileum. This affected segment contrasts with the margin of normal calibre small bowel (arrow). (B) The intraluminal macroscopic morphology of the resected intestinal segment revealed by a longitudinal incision. This demonstrated diverticula (arrowheads), area of mucosal ulceration (arrow), and mural thickening consisting of bulging yellow fat of submucosal lipomatosis (circle pointer). (C) Low-powered photomicrograph of hematoxylin- and eosin-stained sections. This demonstrates infiltration of the thickness of the small bowel wall with numerous adipocyte cells (AC). (D) High-powered photomicrograph of hematoxylin- and eosin-stained sections. Magnification of the boxed area from panel $\mathrm{C}$ confirms the submucosal location of the adipocyte (AC) infiltration.

Histopathological examination of the resected small intestinal segment demonstrated benign submucosal lipomatosis with no evidence of malignancy (Figure 2C,D). The mesenteric lymph nodes showed reactive lymphoid hyperplasia and a few large irregular germinal centres. Incidental intrafollicular neoplasia ("in situ" follicular cell lymphoma) which was evident in several small germinal centres, exhibited strong staining for BCL-2 and CD10 positive cells.

The patient's postoperative recovery was complicated by a microscopic anastomotic leak, causing an asymptomatic gross pneumoperitoneum. However, there were no collections or systemic symptoms. This was managed with intravenous antibiotics. The patient was discharged 2 weeks postoperatively after his presenting symptoms ceased. Importantly, the repeat CT scan at this stage no longer demonstrated lymphadenopathy or abnormalities in the small intestine. At his 6-month follow-up, the patient remained asymptomatic.

\section{Discussion}

Small bowel lipomas are generally submucosal and solitary $[4,15-17,29]$. Although cases of multiple lipomatosis have been reported, the length of involvement and the 
confluent nature of the lipomatosis in our patient makes his case rare in the literature [19]. Only one other case of confluent small bowel lipomatosis has previously been reported in a 39-year-old female. The length of bowel involvement in that case was reported to be $250 \mathrm{~cm}$ [30]. All other reported cases of lipomatosis reported multifocal non-confluent lipomas. Of these, perhaps the most extensive is that of a patient who was reported to have 150 lipomas within a $68 \mathrm{~cm}$ segment of bowel [11]. Intestinal lipomatosis may also be associated with a rare condition, macrodactilia fibrolipomatosis, characterised by intestinal lipomatosis, macrodactyly, and benign fibrofatty infiltration of soft tissues of the distal limbs [22,31,32]. Our patient did not have this condition.

The most common reported complication of intestinal lipomas is small bowel intussusception, although gastrointestinal bleeding has also been reported as a complication $[2,19,20,23]$. The major clinical challenge is in achieving a diagnosis of small intestinal lipomatosis owing to the non-specific nature of clinical presentations. Even upon diagnosis of small bowel lipomas/lipomatosis, establishing the link between this pathology and the presenting symptoms may not be totally certain. Exclusion of other causes often requires extensive investigations. Furthermore, diverticulosis may be associated with small intestinal intussusception [33,34], such as in our case. This association may also have contributed to the patient's symptoms. A CT scan of the abdomen with oral contrast may demonstrate a hypodense lesion or thickening suggestive of a small intestinal tumour. Attenuation values consistent with fat support the diagnosis of small bowel lipomas. Although ultrasound and barium swallow have also been considered useful, especially when the lipomas are palpable, they may not be the best mode of diagnosis $[9,16]$. With our patient, CT scan simply demonstrated widespread hypodense mural thickening of the small intestine which was clearly not diagnostic.

Magnetic Resonance Imaging (MRI), specifically MRE, is now a widely used crosssectional imaging modality for investigation of small bowel pathology. Advantages of enteric imaging with MRI/MRE over CT include lower radiation exposure, better contrast resolution, better contrast medium profiles, better acquisition of images in multiple planes/phases, and the ability to obtain long sequential images over longer timeperiods [35]. Disadvantages of MRI/MRE over CT include higher costs, limited availability, variable image quality, and lower spatial resolution [35]. In the setting of small bowel lipomatosis, MRE also has the advantage of discerning the chemical composition of the mural infiltration. In our patient, the MRE demonstration of macroscopic fat infiltration in the small intestinal wall, indicated by the loss of signal in fat-suppressed images (Figure 1C,D), pointed us in the right direction for diagnosis and management.

Management of small bowel lipomatosis with symptoms or complications is usually by surgical resection [36]. Asymptomatic solitary lesions, where the diagnosis can be confidently made, may be managed expectantly. However, as up to $24 \%$ of small bowel tumours are malignant, small bowel lesion without definitive diagnosis requires resection [1]. Symptoms or complications (such as intussusception or bleeding) are also indications for resection as resection is curative. In contrast to solitary or oligofocal lesions, long confluent lipomatosis or multifocal lipomatosis presents a therapeutic challenge. As in our patient, management will depend on the length of the residual small bowel without lesions. Noninvasive imaging, such as CT and MRI/MRE may over-estimate the extent of disease, like it did in our case. Therefore, our recommendation would be for a laparoscopy or laparotomy to assess the degree of involvement. Provided enough residual small bowel is available, resection of the intestinal segment with lipomatosis is curative.

Concurrently, we also found in situ follicular lymphoma in the mesenteric lymph nodes draining the affected gut segment. In situ follicular lymphomas present with lymph nodes displaying scattered germinal centres with variably-dense populations of BCL-2+ CD10+ monoclonal lymphoid cells that usually have a t $(14 ; 8)$ IGH/BCL-2 translocation without disruption of normal/intact architecture [37]. There is no known association between intestinal lipomatosis and follicular lymphoma, in situ or otherwise. 


\section{Conclusions}

Long segment confluent small bowel lipomatosis as a cause of abdomen pain is rare, with our patient having the longest involved gut segment and the largest number of lipomas on record. More commonly, intestinal lipomatosis occurs as isolated small bowel lipomas, which may present with complications such as intussusception. For symptomatic long segment confluent small bowel lipomatosis, MRI/MRE is a useful non-invasive diagnostic modality, although laparoscopy/laparotomy may be necessary for assessment of the extent of disease. Symptomatic cases should be treated with segmental small bowel resection (with anastomosis) as this is curative.

Author Contributions: S.K.: data curation, formal analysis, software, supervision, validation, visualisation, writing - original draft, writing-review and editing. R.C.: formal analysis, software, writing-review and editing. S.S.: conceptualisation, investigation, methodology, project administration, supervision, validation, writing-review and editing. T.P.: conceptualisation, resources, supervision, visualisation, writing-review and editing. All authors have read and agreed to the published version of the manuscript.

Funding: This research received no external funding.

Institutional Review Board Statement: Not relevant.

Informed Consent Statement: Informed written consent was obtained from the patient involved in this report.

Data Availability Statement: Not applicable.

Conflicts of Interest: None of the authors have conflict of interest or potential conflicts of interest. None of the authors have financial or other interests in any "product or distributor of the product". None of the authors have associations like consultancies, stock ownership, or other equity interests or patent-licensing arrangements.

\section{References}

1. Akagi, I.; Miyashita, M.; Hashimoto, M.; Makino, H.; Nomura, T.; Tajiri, T. Adult Intussusception Caused by an Intestinal Lipoma: Report of a Case. J. Nippon. Med. Sch. 2008, 75, 166-170. [CrossRef] [PubMed]

2. Baron, Y.; Priesack, W.; Sotje, G.; Brix, F.; Scheunemann, C. Hemorrhagic jejunal lipoma with intermittent intussusception. Eur. J. Radiol. 1996, 22, 123-125. [CrossRef]

3. Eyselbergs, M.; Ceulemans, L.J.; De Bontridder, S.; Vanhoenacker, F.; Van Overbeke, L.; Quanten, I.; Jacomen, G.; Snoeckx, A. Ileocolic intussusception due to lipomatosis of the ileum: A common complication of a rare clinical entity. JBR-BTR 2014, 97, 36-38. [CrossRef]

4. Gao, P.-J.; Chen, L.; Wang, F.-S.; Zhu, J.-Y. Ileo-colonic intussesception secondary to small-bowel lipomatosis: A case report. World J. Gastroenterol. 2014, 20, 2117-2119. [CrossRef]

5. Lucas, L.C.; Fass, R.; Krouse, R.S. Laparoscopic Resection of a Small Bowel Lipoma with Incidental Intussusception. J. Soc. Laparoendosc. Surg. 2010, 14, 615-618. [CrossRef]

6. McKay, R. Ileocecal Intussusception in an Adult: The Laparoscopic Approach. J. Soc. Laparosc. Surg. 2006, 10, $250-253$.

7. Moues, C.M.; Steenvoorde, P.; Viersma, J.H.; van Groningen, K.; de Bruine, J.F. Jejunal Intussusception of a Gastric Lipoma. Dig. Surg. 2002, 19, 418-420. [CrossRef]

8. Rathore, M.A.; Andrabi, S.I.H.; Mansha, M. Adult Intussusception-A Surgical Dilemma. J. Ayub Med. Coll. Abbottabad 2006, 18,18 .

9. Urbano, J.; Serantes, A.; Hernandez, L.; Turegano, F. Lipoma-induced jejunojejunal intussusception: US and CT diagnosis. Abdom. Imaging 1996, 21, 522-524. [CrossRef]

10. Zissin, R. Enteroenteric intussusception secondary to a lipoma: CT diagnosis. Emerg. Radiol. 2004, 11, 107-109. [CrossRef]

11. Young, T.-H.; Ho, P.; Tang, H.-S.; Hsu, C.-T.; Chao, Y.-C. A Rare Cause of Multiple Intussusceptions: Intense Segmentary Lipomatosis of The Ileum. Am. J. Gastroenterol. 1996, 91, 162-163. [PubMed]

12. Tatsuguchi, A.; Fukuda, Y.; Moriyama, T.; Yamanaka, N. Lipomatosis Of The Small Intestine And Colon Associated With Intussusception In tTe Ileocecal Region. Gastrointest. Endosc. 1999, 49, 118-121. [CrossRef]

13. Karthikeyan, V.S.; Dhanesekar, P.; Sistla, S.C.; Ali, M.S.; Balasubramaniam, G.; Rajkumar, N. Jejuno-jejunal Intussusception Secondary to Small-Bowel Lipomatosis: A Case Report. S. Afr. J. Sci. 2012, 50, 43-44.

14. Lill, M.; Berkeley, B.; Cooper, G. Multiple Lipomatosis-A Rare Cause For Small Bowel Intussusception. N. Z. Med. J. 2007, 120.

15. Suarez-Moreno, R.M.; Hernandez-Ramirez, D.A.; Madrazo-Navarro, M.; Salazal-Lozano, C.R.; Martinez-Gen, R. Multiple intestinal lipomatosis. Case Rep. Cir. Cir. 2010, 78, 163-165. 
16. Fang, S.-H.; Dong, D.-J.; Chen, F.-H.; Jin, M.; Zhong, B.-S. Small intestinal lipomas: Diagnostic value of multi-slice CT enterography. World J. Gastroenterol. 2010, 16, 2677-2681. [CrossRef]

17. Weinberg, T.; Feldman, M., Sr. Lipomas of the gastrointestinal tract. Am. J. Clin. Pathol. 1955, 25, 272-281. [CrossRef]

18. Kaczynski, J.; Hilton, J. Giant lipoma of the small bowel associated with peroforated ileal diverticulum. BMJ Case Rep. 2012, 2012, bcr1220115299. [CrossRef]

19. Pezzoli, A.; Pennazio, M.; Fusetti, N.; Simone, L.; Zelante, A.; Cifala, V.; Sprujevnik, T.; Carella, A.; Gullini, S. Occult intestinal haemorrhage due to lipoma of the small bowel detected with the combined use of the new endoscopic techniques. A report of two cases. Dig. Liver Dis. 2008, 40, 306-309. [CrossRef]

20. Bilgic, Y.; Hasan, B.A.; Yildirium, N.; Alatas, O.; Kanat, B.H.; Sahin, A. Familial Abdominal and Intestinal Lipomatosis Presenting with Upper GI Bleeding. Case Rep. Gastrointest. Med. 2015, 2015, 123723. [CrossRef]

21. Jung, H.-R.; Park, W.-H.; Choi, S.-O.; Kwon, K.-Y.; Lee, S.-S. Intestinal chondrolipoma: Uncommon cause of bowel obstruction. J. Pediatr. Sugery 2007, 42, E21-E23. [CrossRef] [PubMed]

22. Kenkare, S.; Ainapurapu, B. Macrodactylia Fibrolipomatosis Presenting as a Small Bowel Obstruction. South. Med. J. 2010, 103, 248-249. [CrossRef] [PubMed]

23. Balmadrid, B.; Gluck, M. Chronic iron deficiancy anemia caused by small-bowel lipoma. Gastrointest. Endosc. 2014, 79, 678-679. [CrossRef] [PubMed]

24. Blakeborough, A.; McWilliams, R.G.; Raja, U.; Robinson, P.J.A.; Reynolds, J.V.; Chapman, A.H. Pseudolipoma of inverted Meckel's diverticulum: Clinical, radiological and pathological correlation. Eur. Radiol. 1997, 7, 900-904. [CrossRef] [PubMed]

25. Ueda, K.; Kitagawa, A.; Oka, Y.; Nakao, K. Multiple Lipomas Of The Ileum With Volvulous. Arch. Surg. 1977, 112, 1144-1145. [CrossRef]

26. Neilson, D.; Wilkinson, N.; Magell, J. Case of Simultaneous Diveticulosis, Lipomatosis and Volvulus of The Small Intestine. Br. J. Surg. 1990, 77, 105. [CrossRef]

27. Lee, B.J.; Park, J.-J.; Joo, M.K.; Kim, J.H.; Yeon, J.E.; Kim, J.S.; Chun, H.J.; Byun, K.S.; Choi, J.H.; Kim, C.D.; et al. A Case of Small-Bowel Intussusception Caused by Intestinal Lipomatosis: Preoperative Diagnosis and Reduction of Intussusception with Double-Balloon Enterospcopy. Gastrointest. Endosc. 2010, 71, 1329-1332. [CrossRef]

28. Komagata, T.; Takebayashi, S.; Hirasawa, K.; Fukawa, T.; Arai, M. Extensive Lipomatosis Of The Small Bowel And Mesentery: CT and MRI Findings. Radiat. Med. 2007, 25, 480-483. [CrossRef]

29. Mayo, C.W.; Pagtalunan, R.J.G.; Brown, D.J. Lipoma of the Alimentay Tract. Surgery 1963, 53, 598-603.

30. Cojocari, N.; David, L. Acute Intestinal Infarction Due to Diffuse Jejunoileal and Mesenteric Lipomatosis in a 39-Year-Old Woman. Am. J. Case Rep. 2020, 21, e922830. [CrossRef]

31. Gates, L.K.J.; Keate, R.F.; Smalley, J.J.J.; Richardson, J. Macrodactylia Fibrolipomatosis Complicated by Multiple Small Bowel Lipomas and Intussusception. J. Clin. Gastroenterol. 1996, 23, 241-242. [CrossRef] [PubMed]

32. Mazziotti, S.; Salamone, I.; Vinci, S.; Pandolfo, A. Macrodactylia Fibrolipomatosis Associated with Multiple small-Bowel Lipomas. Am. J. Roentgenol. 2006, 186, 1195-1196. [CrossRef] [PubMed]

33. Grudzińska, E.; Mrowiec, S.; Pilch-Kowalczyk, J.; Ciupińska, M.; Kusnierz, K. Small Intestinal Intussusception Due to Complicated Giant Jejunal Diverticulosis. Medicina 2021, 57, 116. [CrossRef] [PubMed]

34. Yakabe, S.; Muranaka, T.; Sumii, T.; Takeshita, M.; Yamashita, T.; Tsuruta, S.; Saku, M.; Yoshida, K. Jejunal lipomatosis with diverticulosis: Report of a case. Surg. Today 1998, 28, 846-849. [CrossRef]

35. Amzallag-Bellenger, E.; Oudjit, A.; Ruiz, A.; Cadiot, G.; Soyer, P.A.; Hoeffel, C.C. Effectiveness of MR Enterography for the Assessment of Small-Bowel Diseases beyond Crohn Disease. RadioGraphics 2012, 32, 1423-1444. [CrossRef]

36. Farkas, N.; Wong, J.; Bethel, J.; Monib, S.; Frampton, A.; Thomson, S. A systematic review of symptomatic small bowel lipomas of the jejunum and ileum. Ann. Med. Surg. 2020, 58, 52-67. [CrossRef]

37. Pillai, R.K.; Surti, U.; Swerdlow, S.H. Follicular lymphoma-like B cells of uncertain significance (in situ follicular lymphoma) may infrequently progress, but precedes follicular lymphoma, is associated with other overt lymphomas and mimics follicular lymphoma in flow cytometric studies. Haematologica 2013, 98, 1571-1580. [CrossRef] 